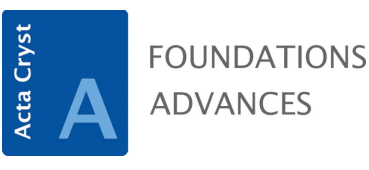

ISSN 2053-2733
Keywords: book reviews; aperiodic crystals; modulated phases; quasicrystals.

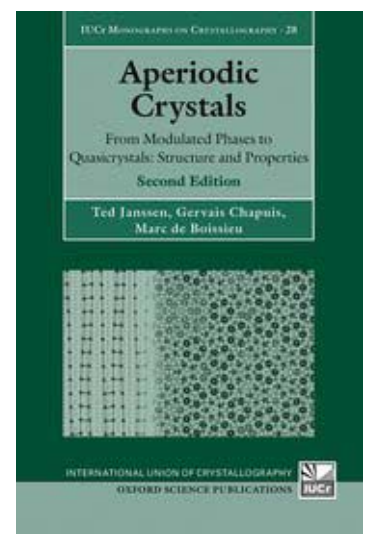

(C) 2018 International Union of Crystallography

\section{Aperiodic Crystals. From Modulated Phases to Quasicrystals: Structure and Properties. Second edition. By Ted Janssen, Gervais Chapuis and Marc de Boissieu. Oxford University Press, 2018. Pp. 560. Price GBP 45.00 (paperback). ISBN 9780198824442.}

\author{
Walter Steurer*
}

Laboratory of Crystallography, ETH Zurich, Wolfgang-Pauli-Strasse 10, CH-8093 Zurich, Switzerland. *Correspondence e-mail: walter.steurer@mat.ethz.ch

Books on scientific areas that are not yet fully explored and understood always reflect in one way or the other the expertise and personal view of the authors. The selection of topics and the way they are presented are influenced by their research areas and interests. The second edition of the book Aperiodic Crystals. From Modulated Phases to Quasicrystals is no exception. There exist already a number of monographs on the different classes of aperiodic crystals (ACs), incommensurately modulated phases (IMPs), composite crystals (CCs) and quasicrystals (QCs). The present book Aperiodic Crystals tries to present all three classes together in a unified manner, showing what ACs have in common, and in which ways they differ from one another. This is the big plus of the book. Compared with the monographs, the focus is less on crystallography and structures but more on dynamics and physical properties.

By definition, an ideal AC is characterized by a discrete pure point Fourier spectrum supported on a $\mathbb{Z}$-module of rank $n>d$, with $d$ the dimension of the AC. Consequently, their structures and dynamics can be described within the framework of the higherdimensional approach. However, there are fundamental differences concerning their relationships to periodic reference lattices: IMPs can be seen as incommensurate modulations and CCs as intergrowths of $d$-dimensional (dD) periodic structures; in contrast, QCs can be described in $\mathrm{dD}$ space based on their underlying tilings only. Furthermore, all known stable QCs exhibit non-crystallographic rotational diffraction symmetry $(N=5,8,10,12)$. This would not be possible for IMPs and CCs due to the existence of their periodic average structures. Also, our understanding of the three classes of ACs is on different levels. While the physics of IMPs and CCs is quite well understood nowadays, this is certainly not the case for QCs. In particular, there are still many open questions concerning their formation, growth and stability (see also Steurer, 2018).

Overall, the book has grown by 66 pages, from 466 pages in the first edition [see also the book review by Clarke (2008)] to the current 532 pages. A few of these new pages are dedicated to more recent developments in AC research, topics such as superspace and crystal chemistry, soft QCs or the natural QCs found in meteorites. A few pages are taken by a couple of exercises and solutions, which have been added to chapters 1,2 and 3 . A little bit disappointing is the part dealing with QC structures and their analysis. There are just three examples discussed in detail, the same as in the first edition. Since more than 50 QCs and a similar number of approximants have been studied to date, a table listing them together with some characteristic parameters would have been quite useful for showing their abundance and diversity. I have not found any hint of the problem of multiple diffraction that can strongly bias the weak reflections in a QC diffraction data collection. The weak reflections are crucial for determining the long-range quasiperiodic order. Although a new section on the growth of QCs has been added in the second edition, the most recent growth simulations (e.g. Kuczera \& Steurer, 2015) have not been mentioned, for instance. In my opinion, the ten years of QC research between the first (2007) and second (2018) editions, reflected in $\sim 2500$ publications, did not find an adequate representation in the new edition. 
There are a couple of minor flaws: the discovery of X-rays is attributed to Max von Laue (1912) instead of to W. C. Röntgen (1895). Fig. 2.5 does not show the diffraction pattern of a decagonal QC but of a phase that is quasiperiodic in one direction only, and periodic in the others. Consequently, the intensity distribution shows pseudo-tenfold symmetry only. Symbols are not always used in a consistent manner: Roman and italic fonts in space-group symbols change in some cases from section to section, and rarely follow the notation given in the International Tables for Crystallography; lattice parameters are in some cases given as $a, b, c$ and in other cases as $a_{i}$;
$\mathbf{H}$ includes the factor $2 \pi$ sometimes and sometimes not; the one-dimensional diffraction vector is denoted $\mathbf{H}$, and so is the Hamiltonian etc. Anyway, these are minor flaws that do not diminish the quality of this otherwise excellent book. If you do not already own the first edition of Aperiodic Crystals, I strongly recommend you buy the second edition.

\section{References}

Clarke, R. (2008). Crystallogr. Rev. 14, 85-87.

Kuczera, P. \& Steurer, W. (2015). Phys. Rev. Lett. 115, 085502. Steurer, W. (2018). Acta Cryst. A74, 1-11. 